\title{
The cAMP receptor CAR4 regulates axial patterning and cellular differentiation during late development of Dictyostelium
}

\author{
John M. Louis, Gail T. Ginsburg, and Alan R. Kimmel \\ Laboratory of Cellular and Developmental Biology, National Institute of Diabetes and Digestive and Kidney Diseases, \\ National Institutes of Health, Bethesda, Maryland 20892 USA
}

\begin{abstract}
Pseudoplasmodia of developing Dictyostelium are organized with anteroposterior polarity. We have isolated $C A R 4$, the gene for a new cell-surface, G protein-linked cAMP receptor. CAR4 mRNA is initially expressed during tip elongation and continues to accumulate into culmination. CAR4 is maximally expressed in pseudoplasmodia anteriors which are centers for extracellular cAMP signaling and for organization of cellular patterning. Although car 4 null cells progress unperturbed through early development, they exhibit major patterning aberrations as the anteroposterior axis becomes established. Prestalk gene expression is significantly reduced in car4 nulls, whereas prespore-specific markers are overexpressed and detected in zones normally restricted to prestalk cells. Patterning defects are similarly apparent in terminally differentiated fruiting bodies. Our results show that cAMP signaling is required for pattern formation and cellular differentiation during late Dictyostelium development.
\end{abstract}

[Key Words: cAMP; differentiation; receptors; gene expression]

Received May 17, 1994; revised version accepted July 18, 1994.

Early in development, Dictyostelium initiate a communication system based on the production of secreted, extracellular cAMP (Kimmel and Firtel 1991). Through receptor-mediated responses to extracellular cAMP, cells aggregate and differentiate into progenitor prestalk and prespore cells. Initially, these differentiated cells appear to be distributed randomly throughout loose aggregation mounds, but as the mounds become more defined, the cells sort into distinct populations (Williams et al. 1989; Esch and Firtel 1991). The differentiated cell types can be distinguished by the expression of specific marker genes. Prestalk cells are defined by the expression of two different extracellular matrix proteins, $\mathrm{EcmA}$ and $\mathrm{EcmB}$ (Jermyn et al. 1987). The ecm A-expressing prestalk cells migrate to the upper surface of the mound and form a tip while $e c m B$-expressing cells localize to both the tip and the base of the mound (Williams et al. 1989). The prespore cells, which express the 14-E6 and $\cot B$ genes, among others, comprise the major portion of the body of the aggregate (Williams et al. 1989; Esch and Firtel 1991; Fosnaugh and Loomis 1993).

The prestalk tip of the aggregate is the organizing center for patterning and the primary site of cAMP signaling (Raper 1940; Bonner 1949; MacWilliams 1984). Extension of the tipped aggregate into a finger-like projection transforms the patterned mound into an elongated pseudoplasmodium or migrating slug. A cell fate model for development of Dictyostelium indicates that the cellular pattern of the tipped aggregate is translated into an anteroposterior (AP) axis during slug formation (Williams 1991). The tip of the aggregate forms the anterior $20 \%$ of the slug and continues as the cAMP signaling center. The posterior $80 \%$ is primarily derived from the prespore cells of the body of the aggregate.

The prestalk cells form discrete cell populations based on marker gene expression and spatial location within the slug. Prestalk cells in the front half of the anterior region express ecmA at high levels and are called prestalk A (pstA) cells, whereas the prestalk 0 (pst0) cells form the back half of this region and express $e c m A$ at lower levels (Early et al. 1993). Prestalk cells within the core of the anterior region that express ecm $B$ as well as ecm $A$ are called prestalk $\mathrm{AB}$ (pstAB) cells (Gaskell et al. 1992). Although the posterior of the slug is comprised mostly of prespore cells, an anterior-like cell (ALC) population is scattered randomly among the prespore cells (Sternfield and David 1982) and some of the ALCs can exchange with pst 0 cells during slug migration (Early et al. 1993). These ALCs of the posterior may express ecm $A$ and/or ecmB, but not prespore markers (Gaskell et al. 1992). The rear-guard cells of the pseudoplasmodium primarily express ecmB but not ecm $A$. Some of these cells become deposited on the developmental surface as the slug migrates.

When slug migration arrests, a second upright structure forms that retains the cellular pattern (Williams 1991). Culmination, the terminal differentiation into the mature fruiting body, begins with the anterior pstAB 
cells differentiating into stalk cells and migrating through the prespore region toward the base. As the stalk tube develops, it pushes the prespore mass upward. The ALCs and the pst 0 cells form the upper and lower cups that surround the prespore cells and support them as they differentiate into spores (Early et al. 1993). Thus, the cellular pattern of the mature fruiting body can be traced from the AP polarity initially established after aggregation.

cAMP signaling is utilized during many stages of the developmental cycle of Dictyostelium (Kimmel and Firtel 1991; Johnson et al. 1992a; Saxe et al. 1993). Signaling requires the stimulation of cell-surface receptors for cAMP and the subsequent activation of $G$ protein-linked effector pathways. We have previously isolated the genes for three cAMP receptor subtypes, CAR1, CAR2, and $C A R 3$, and have provided evidence for a fourth, CAR4 (Saxe et al. 1991a; Johnson et al. 1993; Saxe et al. 1993). CAR1 encodes a high-affinity cAMP receptor that is expressed at maximal levels during early development and at reduced levels after aggregate formation (Saxe et al. 1991a; Johnson et al. 1992b). Cells that are unable to express CAR1 do not initiate cAMP signaling, nor do they aggregate or differentiate (Klein et al. 1988; Sun and Devreotes 1991).

CAR2 has a lower affinity for cAMP than does CAR1 and is maximally expressed in the prestalk cells of tipped aggregates (Johnson et al. 1992b; Saxe et al. 1993). Cells carrying a gene disruption of $C A R 2$ aggregate normally but do not form tips of differentiated prestalk cells, nor do they regulate cell type-specific gene expression appropriately (Saxe et al. 1993). We have suggested that CAR2 organizes the pattern of the aggregation mound.

We have now isolated CAR4, which we suggest to be the final member of this cAMP receptor gene family (Saxe et al. 1993). CAR4 mRNA is initially expressed during tip elongation and continues to accumulate into culmination. Throughout development, CAR4 is preferentially expressed in tips and slug anteriors, centers for cAMP signaling and pattern organization. Through the use of homologous recombination to disrupt CAR4 and the introduction of markers to detect cell type-specific patterns of expression, we have studied the development of Dictyostelium mutants that cannot produce CAR4. As expected, car4 mutants initially develop normally; aggregation and tip formation proceed without a phenotypic change. However, the resulting slugs exhibit an increasingly aberrant cellular pattern as development continues. Levels of prestalk and prespore gene expression are abnormal, and pattern formation is disrupted. Markers for certain prestalk cells appear to be underrepresented, and prespore patterns of expression are additionally observed in regions normally restricted to prestalk cells. These slugs migrate poorly and form culminant structures at low frequency. The few resulting fruiting bodies exhibit abnormal cellular patterns that can be traced from those of the aberrant slugs.

We conclude that cAMP signaling is required throughout the entire developmental program of Dictyostelium. In particular, CAR4 regulates cell type-specific gene ex- pression, cytodifferentiation, and pattern formation during the late stages of development and it is critical for establishing the predominant AP axial polarity that will define the terminally differentiated organism.

\section{Results \\ Isolation and characterization of CDNA and genomic sequences of CAR 4}

Using probes of CAR1 that cross-hybridize with CAR2 and $C A R 3$, we suggested previously the presence of a gene for a fourth cAMP receptor subtype in Dictyostelium (Saxe et al. 1991a, 1993). We confirmed the existence of CAR4 through the isolation of cDNA and genomic recombinant clones and its functional expression in growing Dictyostelium (see below).

The derived amino acid sequence of CAR4 is depicted in Figure 1A. Our sequence data for CAR4 extend 1251 nucleotides upstream of transcription initiation and indicate the presence of two introns: One (113-bp) is located in the 322-nucleotide $5^{\prime}$-untranslated region, and the other $(112 \mathrm{bp})$ interrupts the protein-coding region (Fig. 1A). RNA and protein-coding regions were identified by comparison of genomic and CDNA sequences and confirmed by primer extension and $\mathrm{S}_{1}$-nuclease protection studies.

Growing Dictyostelium express only $\sim 5000$ cAMP receptors per cell; these numbers increase $>10$-fold during development. To confirm that the predicted CAR4 protein is a surface receptor for CAMP, we demonstrated that its expression in growing cells substantially increases the number of cAMP-binding sites. The CAR4 cDNA sequence was fused to a Dictyostelium actin promoter that permits expression of linked genes in growing cells. The fusion construct was transformed into Dictyostelium, and cell-surface binding for cAMP was measured in CAR4-expressing cells and in controls. In three separate transformed cell lines we observed $\sim 225,000$ cAMPbinding sites per cell compared with only 6000 sites for controls grown under identical conditions.

As expected, CAR4 is substantially related in sequence to CAR1, CAR2, and CAR3 (Fig. 1A; Klein et al. 1988; Johnson et al. 1993; Saxe et al. 1993|. Each receptor subtype possesses seven domains enriched in hydrophobic residues that are predicted to form membrane-spanning helices (Klein et al. 1988). This heptahelical motif is common to all members of the $G$ protein-linked receptor superfamily (Dohlman et al. 1991). In addition, there is $\sim 25 \%$ sequence identity ( $\sim 65 \%$ similarity) with the $\sim 70$ amino acids that span transmembrane domains 5 and 6, cytoplasmic loop 3 of CAR4 (Fig. 1A), and corresponding regions of the vertebrate dopamine $\mathrm{D} 2$ and endothelin B receptors.

Comparative sequence analyses of the four cAMP receptor subtypes indicates that they comprise one of the most related groups of $\mathrm{G}$ protein-linked receptors. Overall, their amino acid sequences are $\sim 40 \%$ identical $(\sim 55 \%$ similar), a relatedness that increases to $\sim 55 \%$ identity $(\sim 80 \%$ similarity $)$ when only transmembrane 
Louis et al.

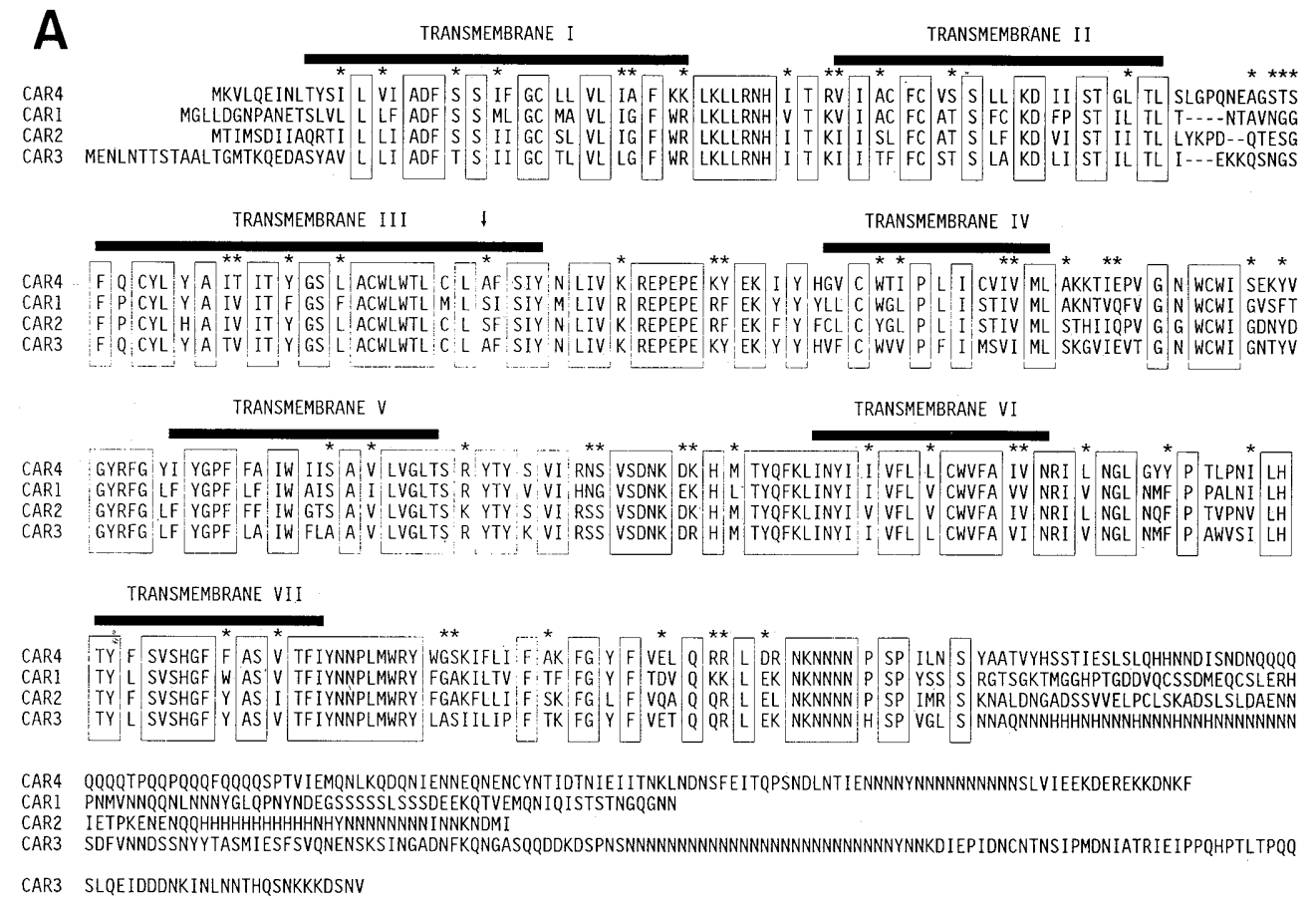

Figure 1. Analyses of derived amino acid sequences of CAR4. $(A)$ The amino acid sequences of CAR4, CAR1, CAR2, and CAR3 with transmembrane domains are indicated according to those predicted for CAR1 (Klein et al. 1988). Amino acid identities for all CAR proteins are enclosed in boxes with similarities indicated by asterisks (*). A single intron of $112 \mathrm{bp}$ is located within the coding region of transmembrane domain 3 of CAR4. This

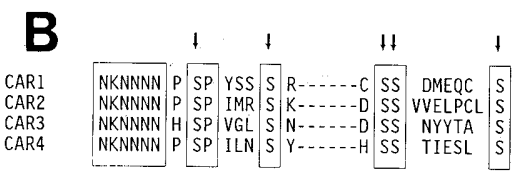
intron position (downward arrow) is identically conserved in CAR1,CAR2, and CAR3

(Saxe et al. 1993). The CAR4 sequence has been deposited in the PIR data base. (B) Identities within the carboxyl-terminal regions are boxed. Arrows indicate potential sites for phosphorylation of CAR1 (Hereld et al. 1994). Dashes denote nonconserved distances between related seryl domains.

and loop domains are compared (Fig. 1A). It is striking that but for a variability of 2 amino acids in intracellular loop 1, the sequence lengths of each domain are identically conserved, as is the location of the single intron in their protein-coding regions (Fig. 1A; Saxe et al. 1993). Nonetheless, the extracellular amino-terminal domains exhibit no sequence homology and, although the terminal intracellular carboxyl domains of CAR2, CAR3, and CAR4 (but not CAR1) possess extended homopolymeric stretches of asparagine, they otherwise have no extended sequence similarity. Apart from the asparagine clusters, each of the receptors is equivalently related to each of the others; $54-60 \%$ identity $(68-71 \%$ identity within transmembrane and loop domains). The multiple clusters of seryl residues in the carboxyl terminus of CAR1, which are sites for ligand-dependent (and possibly ligand-independent) phosphorylation, are similarly organized in the carboxyl termini of CAR2, CAR3, and CAR4 and may represent conserved modification sites involved in their regulation (Fig. 1B; Klein et al. 1985; Hereld et al. 1994). Finally, it should be noted that there are glutamine clusters in the carboxy-terminal domain of CAR4, a feature observed in such diverse proteins in Dictyostelium as the catalytic subunit of protein kinase A and the putative nuclear factor rZIP (Kimmel and Firtel
1985; Shaw et al. 1989; Mann and Firtel 1991; G.T. Ginsburg and A.R. Kimmel, unpubl.).

\section{Temporal and spatial patterns of CAR4 expression}

We used several criteria to analyze the expression of CAR4 during Dictyostelium development. To determine its time course of expression, poly $(\mathrm{A})^{+}$RNA was isolated at major stages during development and hybridized to CAR4 on Northern blots (Fig. 2A). We detect no CAR4 mRNA in growing cells $(0 \mathrm{hr})$, aggregating cells $(5 \mathrm{hr})$, or loose mounds $(10 \mathrm{hr})$. CAR4 is first expressed as mounds elongate and expression is maximal into culmination (12-20 hr; Fig. 2A).

Prestalk and prespore cells isolated from migrating slugs exhibit density differences when separated by Percoll gradient centrifugation (Ratner and Borth 1983). We used these cell populations to examine cell typespecific expression of CAR4. Northern blots clearly demonstrate a preferential (about sixfold) accumulation of CAR4 mRNA in the less dense, prestalk (anterior and anterior-like) cells compared with the denser, prespore (posterior) population (Fig. 2B). Although these data indicate differences in expression levels between the two cells types, they are insufficient to specifically localize 
$\boldsymbol{A}$

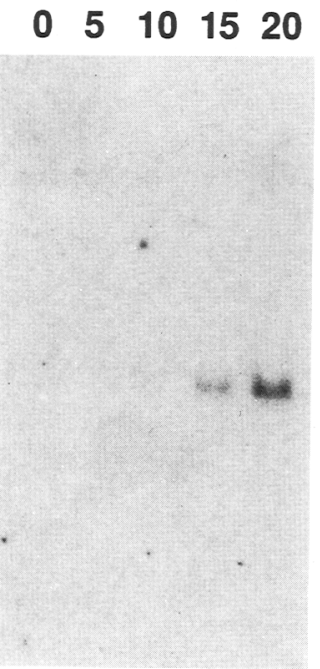

$\boldsymbol{B}$

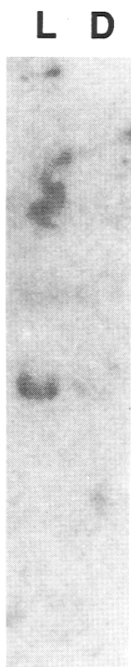

Figure 2. Regulation of CAR4 mRNA expression. (A) Expression during development. Wild-type NC-4 cells were grown on bacteria, harvested in logarithmic phase (0), washed, plated on an agar surface, and allowed to synchronously proceed through development. At 5-hr intervals, cells were collected and RNA was isolated. Equivalent amounts of RNA were size separated on denaturing gels and blotted for hybridization to CAR4. (B) Expression in density-gradient separated cell types. Developed slugs were physically disrupted and separated by density on preformed Percoll gradients. RNA was isolated from the two cell populations and analyzed by gel blot hybridization to CAR4. (L) lighter cell (prestalk) fraction; (D) denser cell (prespore) fraction.

CAR4 expression within the developing organism. Therefore, we fused a 1638-bp region of CAR4 comprising the transcription initiation site (1251 bp of upstream and 387 bp of transcribed sequences) to the Escherichia coli lacZ gene. This construct was transformed into Dictyostelium to localize CAR4 expression during development by in situ cytological staining for $\beta$-galactosidase.

No significant $\beta$-galactosidase activity is detected in cells until tip elongation (Fig. 3A). These data are consistent with the temporal regulation of CAR4 detected by developmental Northern blot analysis (Fig. 2A). There is clearly greater $\beta$-galactosidase activity in the anterior prestalk cells, the cAMP signaling centers of slugs (Fig. 3B), and in prestalk and ALC-derived structures / the stalk tubes and the upper and lower cups; Fig. 3C) than in prespore/spore cells. However, upon prolonged incubation, all regions and cells appear to stain. These data are in agreement with the hybridization experiments that indicate a preferential, but not exclusive, expression of CAR4 in prestalk cells (Fig. 2B). Similar constructs deleted to -837 of upstream sequence yielded identical patterns of staining, suggesting that these model in vivo expression of CAR4.

\section{Gene disruption of CAR4}

Gene disruption by homologous recombination has proven to be effective for functional analyses in Dictyostelium, a strategy we utilized to examine the development of strains that lack a functional CAR4 protein. We inserted the selectable marker THY1 into a region of $C A R 4$ that is conserved among all four cAMP receptor subtypes (Klein et al. 1988; Johnson et al. 1993; Saxe et al. 1993) and has proven effective for the functional disruption of CAR2 (Saxe et al. 1993). Disrupted CAR4 plasmid was electroporated into thy $1^{-}$Dictyostelium, and transformants were selected for growth in the absence of thymidine. Double crossover recombinants were confirmed by polymerase chain reaction (PCR) analysis and Southern blot hybridization. In the positive clones, the $C A R 4$ gene is replaced by a single disrupted copy containing the THY1 marker and migrates with altered mobility as revealed by genomic Southern blot analysis. No additional inserts were detected.

Next, we examined the ability of car4-disrupted cells to develop. No obvious differences were observed between parentals and car4 nulls through early developmental stages. However, examination of car4 ${ }^{-}$slugs revealed considerable abnormalities (Fig. 4A). Control (JH10) slugs are capable of extended migration. The observed trails represent deposited cellular and noncellular material. In contrast, car4 mutant slugs are deformed and extremely impaired in migration. This is most obvious when the migratory trails of $\mathrm{JH} 10$ and car4 $4^{-}$slugs are compared (Fig. 4A). As development proceeds, more aberrant structures become evident. Figure 4B shows a typical mature fruiting body of $\mathrm{JH} 10$, with two representative structures of developed car4- cells. In general, these mutant fruiting bodies are more squat than wildtype cells, with an apparent increase in the ratio of sorus mass to stalk length. It should also be emphasized that whereas normally $\sim 95 \%$ of $\mathrm{JH} 10$ cells complete development, we routinely observe fewer than $25 \%$ terminal structures in equivalent platings of car4 nulls on agar surfaces.

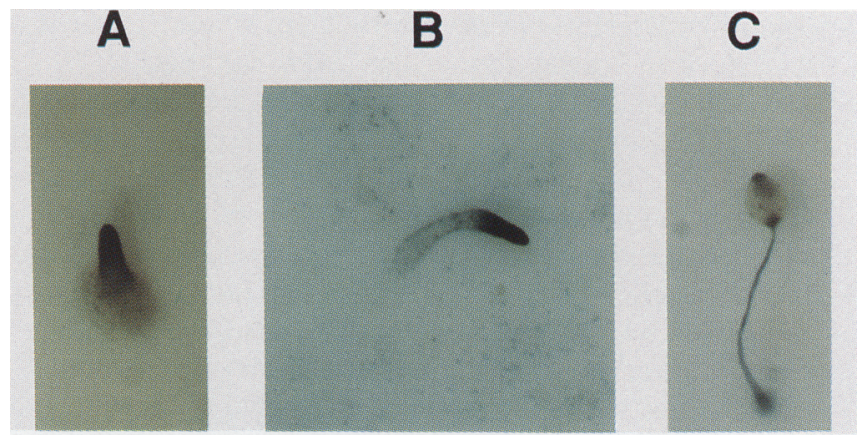

Figure 3. Spatial expression of $C A R 4$ during development. $\beta$-Galactosidase staining of developed structures carrying the $C A R 4$ promoter/lacZ fusion. $(A)$ Tipped aggregate; $(B)$ slug; $(C)$ mature fruiting body. 
Figure 4. Developmental morphologies of control and car4- cells. JH10 and car4 ${ }^{-}$ cells were harvested in logarithmic phase, washed, plated on filter pads, and allowed to synchronously proceed through development. (A) $\mathrm{JH} 0$ and car4 $4^{-}$cells at $16 \mathrm{hr}$ of development; $(B) \mathrm{JH} 10$ and car $4^{-}$cells at $28 \mathrm{hr}$ of development.

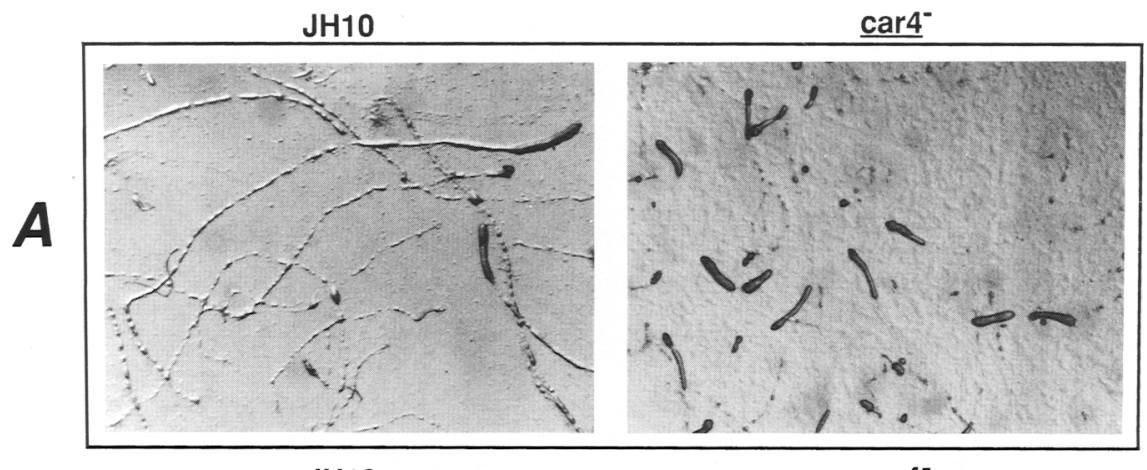

$\mathrm{JH} 10$

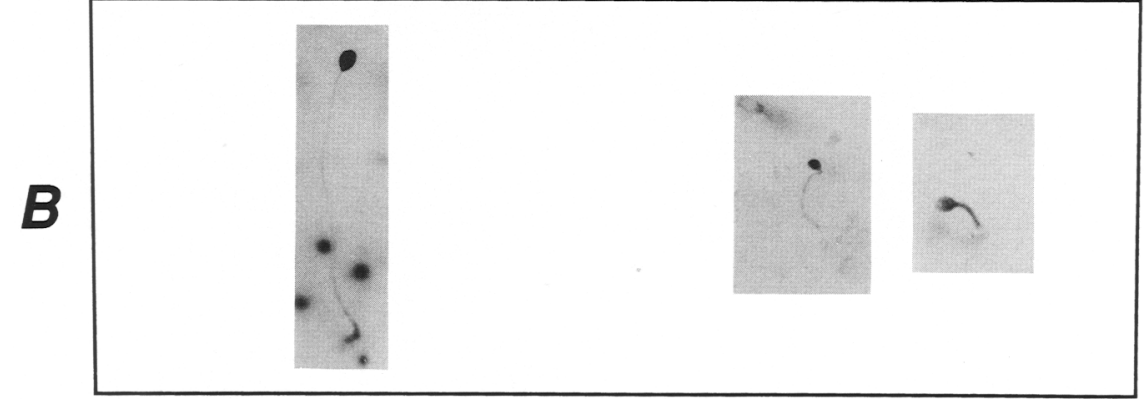

car4- car4 ${ }^{-}$cells exhibit defects in cell type-specific gene expression

We have followed cell type-specific gene expression throughout the development of car4 $4^{-}$and parental control strains. Figure 5A shows results of developmental RNA blots probed with prestalk markers ecmA and $e c m B$ or with the prespore markers $14 E-6$ and $\cot B$. Tip formation for both parental and mutant axenic strains occurred on agar surfaces simultaneously, $\sim 3-4 \mathrm{hr}$ sooner than was observed on filter pads for the wild-type NC-4 cells used for Figure 2A. Both ecmA and ecmB mRNAs appear to be induced at the appropriate stage of development, but their levels of accummulation are severely impaired when compared with controls; only part of this reduction can be attributed to a loss of developmental synchrony. In parallel with the decrease in prestalk expression, we see more than fivefold overexpression of the prespore markers $14 E-6$ and $\cot B$.

The expression of prespore genes is dependent on receptor activation by extracellular CAMP /Schaap and van Driel 1985; Gomer et al. 1986; Haribabu and Dottin 1986; Oyama and Blumberg 1986; Kimmel 1987). Thus, a differential accumulation of cAMP in car4 ${ }^{-}$cells, as compared with controls, could potentially cause the overexpression of prespore markers. However, we observed a similar level of overexpression of prespore markers in suspension cultures incubated with saturating concentrations $(>300 \mu \mathrm{M}$ ) of exogenous cAMP (Fig. $5 \mathrm{~B})$, indicating that the effect of the car4 mutation on prespore gene expression is not simply an alteration in the accumulation of extracellular cAMP.

\section{Abnormal cellular differentiation and patterning in car4 nulls}

To ascertain the role(s) of CAR4 in cytodifferentiation more clearly, we examined the spatial patterns of expression of cell type-specific genes in the car4 disruptants. We utilized fusions of the E. coli lacZ gene to the promoters of the prestalk genes $e c m B$ and $e c m A$ (Williams et al. 1989) and of the prespore gene $\cot B$ (Fosnaugh and Loomis 1993), and examined expression of these constructs by performing cytological staining for $\beta$-galactosidase in whole organisms.

Control cells expressing $e c m B$ during aggregation are localized at the base of the mound and the core of the tip, the presumptive stalk tube. As the mound elongates into a slug, ecmB expression is concentrated in the anterior pstAB cells, in some of the ALCs that are distributed diffusely through the posterior region, and in the rearguard zone (Fig. 6A). The posterior staining is pronounced during slug migration and is detected in cells sloughed into the slime trail (Fig. 4A and 6A). A dramatic difference in expression of ecmB is seen in car $4^{-}$cells. The decrease in $e c m B$ expression detected by developmental Northern blots (Fig. 6A) is manifested as reduced expression in the anterior zone of the slug and as an almost complete absence, even after extended slug migration, of $e \mathrm{~cm} B$ expression in the posterior, a region that would eventually give rise to the lower cup supporting the spore mass of the mature fruiting body. Consistent with this, we observe no staining of the lower cup of the most normal looking car4 null fruiting bodies (Fig. $6 \mathrm{~B})$, although in very deformed car4 $4^{-}$fruiting bodies, 
A
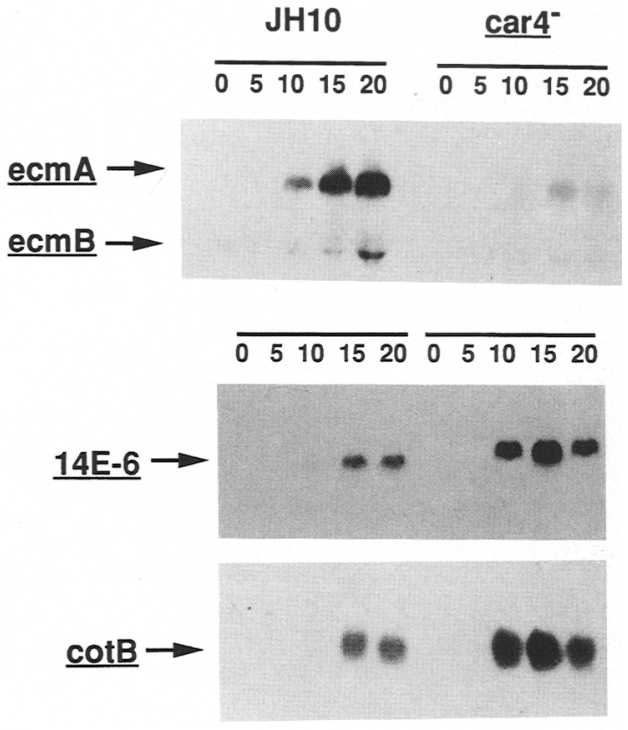

B

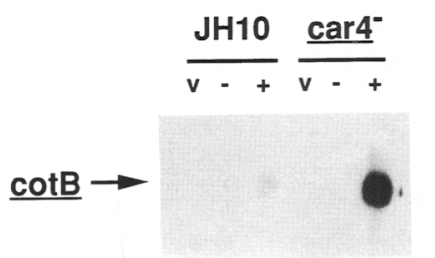

Figure 5. Expression of cell type-specific genes in control and car4 $4^{-}$cells. (A) $\mathrm{JHIO}$ and $\mathrm{car}^{-}$cells were harvested in logarithmic phase (0), washed, plated on an agar surface, and allowed to synchronously proceed through development. Developmental Northern blots containing equivalent amounts of RNA per lane were hybridized to probes for the prestalk genes ecm $A$ and $e c m B$, and to the prespore genes $14 E-6$ and $\cot B$ (Fig. 2A). (B) $\mathrm{JH1O}$ and $\mathrm{car}^{-}$cells were harvested in logarithmic phase (V), washed, and differentiated in liquid culture for $10 \mathrm{hr}$ in the absence $(-)$ or presence $(+)$ of cAMP (Materials and methods). Equivalent amounts of RNA were hybridized on Northern blots to a probe for the prespore gene $\cot B$.

ecmB-expressing cells appear to be distributed ubiquitously.

Expression of ecmA is characteristic of a different prestalk cell population than is expression of $e \mathrm{~cm} B$. ecm $A$ expression is strongest in the pstA cells in the anterior region but weaker in the pst 0 zone and in the ALCs of the slug posterior (Fig. 7A). As was observed with $e c m B$ expression, we see a significant decrease in ecmA expression in posterior regions of slugs and in lower cups of culminants of car4 nulls (Fig. 7B). The expression data for $e c m A$ and $e c m B$ suggest an involvement of CAR4 in the differentiation and/or patterning of the ALCs, and perhaps psto cells as well.

A clearer example of aberrant patterning in car 4 nulls is seen with the prespore marker $\cot B$. In wild-type cells, $\cot B$ is normally expressed only in the posterior regions of slugs and early culminants, and in the spores of fruiting bodies (Fig. 8). Under the described conditions of development, these zones of expression are very specific in wild-type cells, as we do not observe expression of $\cot B$ in the anterior, prestalk regions of slugs or in stalk tubes (Fig. 8). In contrast, the car4 nulls appear to be less restrictive in their spatial distribution of prespore gene expression. $\cot B$ expression is very evident in anterior regions of first fingers (Fig. 8A) and early slugs, in stalks of mature fruiting bodies (Fig. $8 \mathrm{~B}$ ), and even in the tips of developmentally arrested aggregates. These zones are normally absent of prespore gene expression. The only region of the $\mathrm{car}^{-}$slugs that was devoid of significant $\beta$-galactosidase expression was the extreme anterior tip (Fig. 8A).

Two possibilities exist for the observed expression of $\cot B$ in the anterior zones of car 4 disruptants. Prestalk cells of slug anteriors may additionally express prespore (posterior) markers or, alternatively, cell type patterns of expression may remain specific but prespore cells may cross the normal prestalk boundary and not be restricted to the posterior region of slugs. In the former situation, prestalk cells expressing $\cot B$-driven $\beta$-galactosidase would differentiate into stainable stalk cells; in the latter, blue cells present in the stalk tube would derive from prespore cells that had mislocalized to the slug anterior. These cells could be developmentally arrested and may not differentiate into stalk cells. Therefore, we examined the morphology of stained cells in the stalks of car4 nulls carrying the $\cot B / 1 a c Z$ marker by phase microscopy at high magnification. Cells within the stalk tubes of car 4 nulls clearly stain for $\beta$-galactosidase, but this activity is absent in the true cuboidal-shaped stalk cells (Fig. 8C). Only small, irregular cells of the stalk appear blue. Obvious staining of stalk cells (and of upper and lower cups) is observed in an ecmA/lacZ-marked wild-type strain, whereas stalks of wild-type cells carrying the $\cot B / 1 a c Z$ marker do not stain detectably (Fig. 7 and 8). These data indicate that although prespore-marked cells of the anterior region eventually become deposited within the stalk tube, they are not immediate precursors of stalk cells. We suggest that expression of a prespore marker in the anterior, prestalk zone of car4- $4^{-}$strains reflects a defect in positioning of prespore cells rather than a defect in gene regulation of prestalk cells.

\section{Discussion}

Development of Dictyostelium is characterized by distinct axial polarity. We have suggested previously that response to extracellular cAMP, as mediated by the cAMP receptors CAR1 and CAR2, regulates pattern formation of the aggregation mound through control of cytodifferentiation and specific cell sorting (Saxe et al. 1993). The temporal regulation of $C A R 3$ is intermediate between that of $C A R 1$ and $C A R 2$, whereas expression of $C A R 3$ is approximately equivalent in all cell types (Saxe et al. 1991a, 1993; Johnson et al. 1993; R. Gollop and A.R. Kimmel, unpubl.). Because $C A R 3$ disruptants yield 
Figure 6. Spatial pattern of expression of $e c m B$ in $\mathrm{car}^{-}$mutants. $\beta$-Galactosidase staining of control $(\mathrm{JH} 10)$ and car4 $4^{-}$cells carrying the $e \mathrm{cmB}$ promoter/lacZ fusion. (A) Slugs; anterior regions are stained most heavily; $(B)$ fruiting bodies. Arrows depict regional differences in staining between strains.

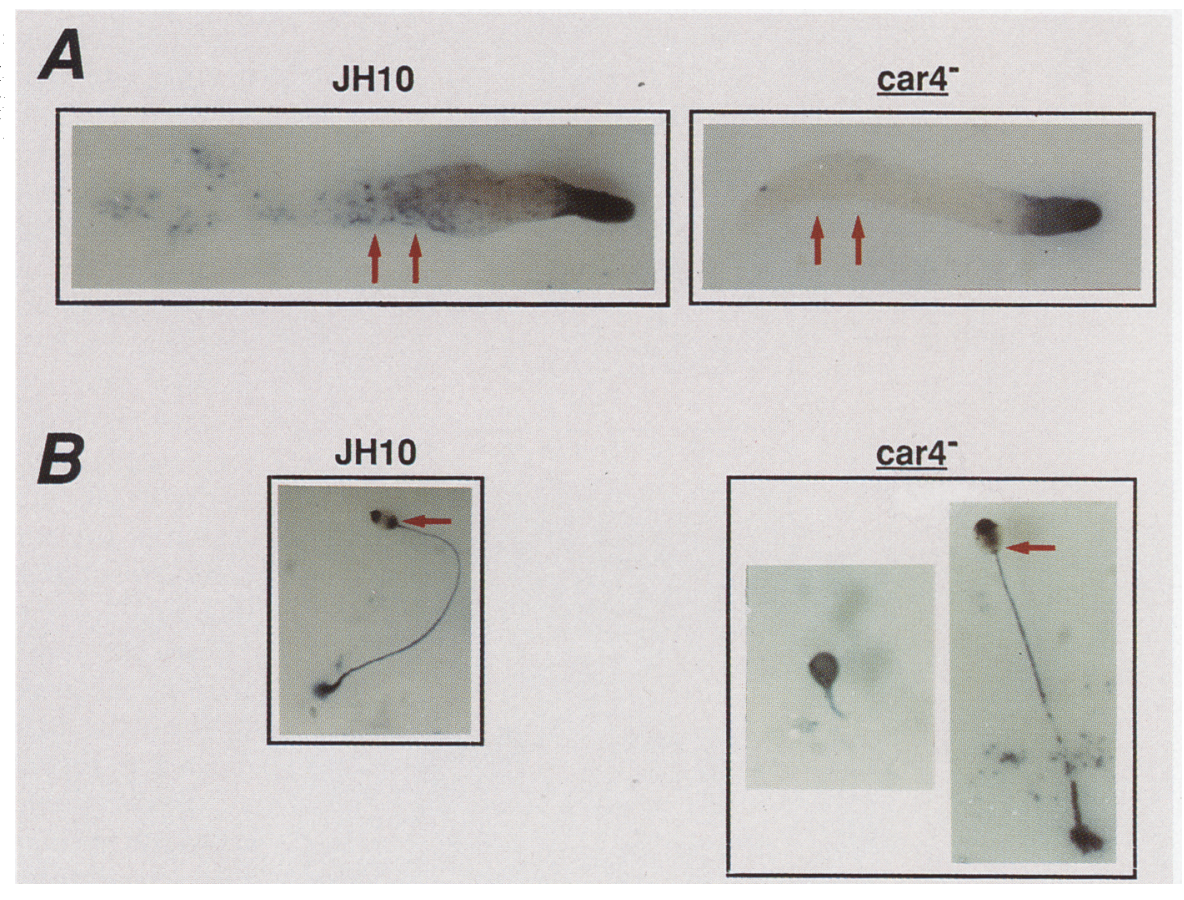

no overt phenotype, we have also suggested that CAR3 may have functions that are partially redundant with those of CAR1 and/or CAR2 (Johnson et al. 1993; Saxe et al. 1993).

By characterizing the function of CAR4, we report here that extracellular cAMP signaling also plays a significant role during the later stages of Dictyostelium development. The absence of CAR4 severely affects the ability of the organism to reorganize itself from a patterned mound into an elongated polarized slug. car $4 \mathrm{mu}-$ tants form slugs and fruiting bodies at reduced frequencies and the few mature structures that do form are significantly compromised in morphology. In particular, gene markers associated with prestalk cells are underexpressed. Although a subpopulation of pstA cells may continue to differentiate and position properly, there is almost no detectable expression in pst0/ALC regions.
The decrease in pst0/ALC expression, coupled with an increase in prespore expression, contrasts with the phenotype of another Dictyostelium mutant, the $g_{\alpha} 4$ null (Hadwiger and Firtel 1992). $G_{\alpha} 4$ encodes a subunit of a heterotrimeric $G$ protein complex in growing cells and in ALCs during development. $g_{\alpha} 4$ nulls form aberrant aggregates that express prespore markers very poorly, suggesting that expression of $G_{\alpha} 4$ during late development is required for differentiation of prespore cells and spores. We have not examined the expression of $G_{\alpha} 4$, but our data indicate that, however small the ALC population is in car 4 mutants, prespore differentiation is not inhibited but may be promoted.

The enhanced expression of posterior prespore markers as well as their unique anterior localization in car4 $4^{-}$ finger structures and slugs may result from multiple phenomena. The reduction in size of the anterior prestalk
Figure 7. Spatial pattern of expression of ecm $A$ in car4 mutants. $\beta$-Galactosidase staining of control $(A x-3)$ and car $4^{-}$cells carrying the ecmA promoter/lacZ fusion. (A) slugs; anterior regions are stained most heavily; $(B)$ culminants/fruiting bodies. Arrows depict regional differences in staining between strains.
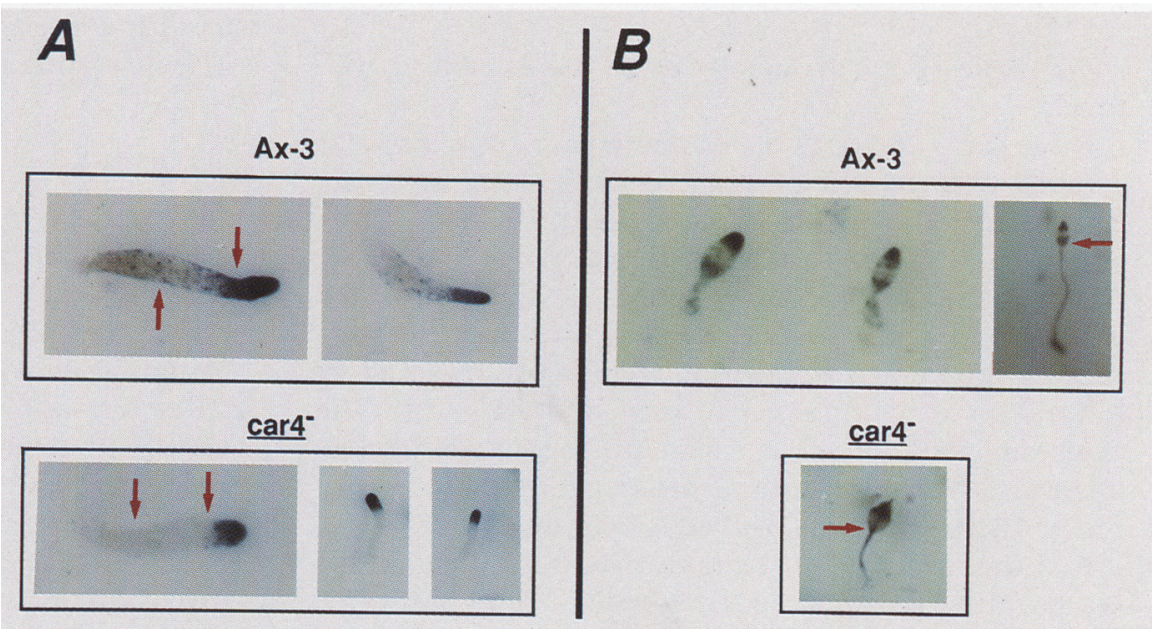


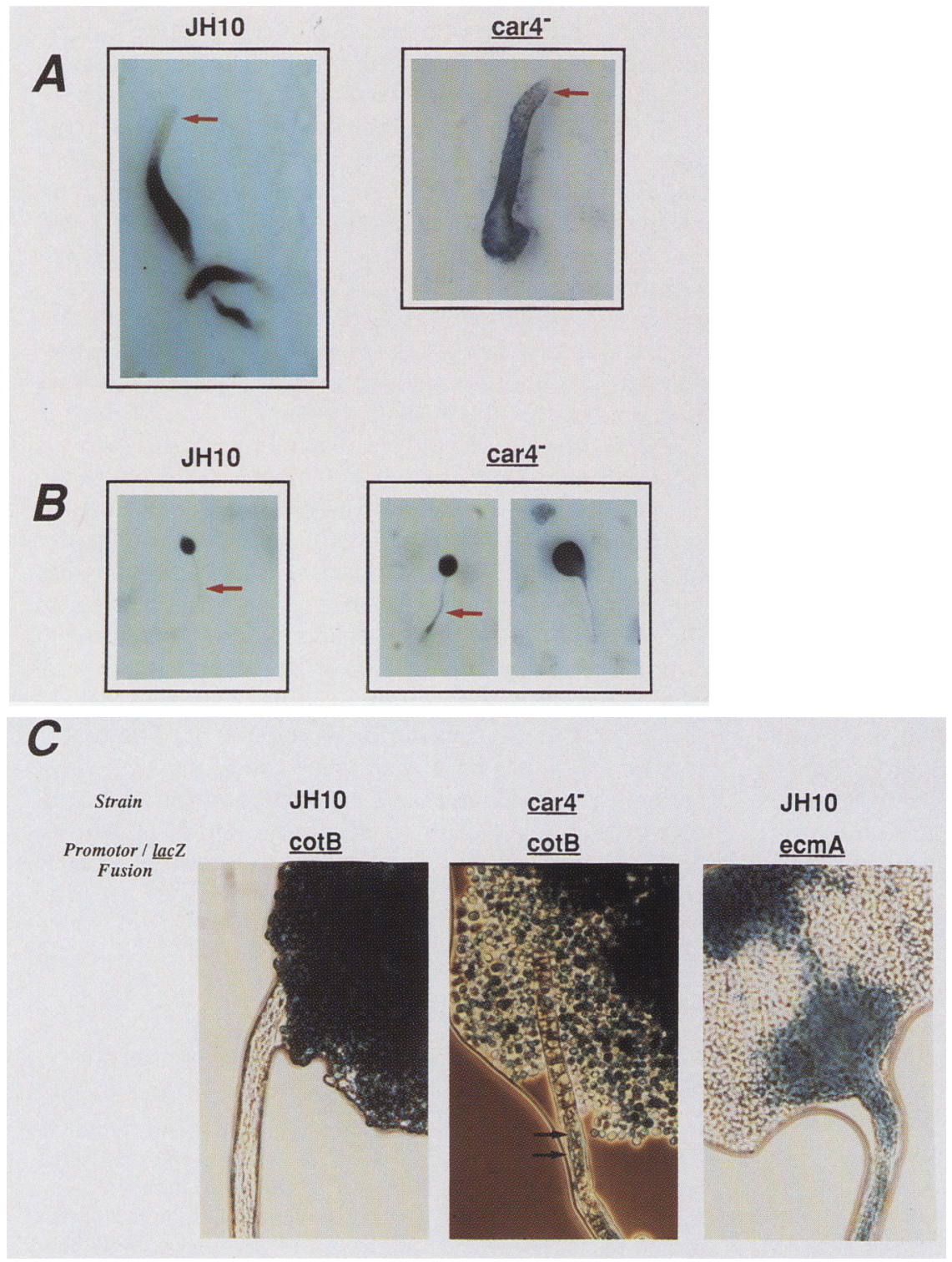

Transmembrane signal control of pattern formation

Figure 8. Spatial pattern of expression of $\cot B$ in car 4 mutants. $\beta$-Galactosidase staining of control $(\mathrm{JHIO})$ and car $4^{-}$cells carrying the $\cot B$ promoter/lac $Z$ fusion. $(A)$ Fingers/ slugs; posterior regions are stained most heavily. Differences in staining intensities between $\mathrm{JH} 10$ and $\mathrm{car}^{-}$strains do not reflect expression levels. Rather, we stained JH10 for extended periods to emphasize its clear, unstained tip. car $4^{-}$cells were understained to demonstrate rapid anterior $\cot B$ expression. (B) Fruiting bodies. Arrows depict regional differences in staining between strains. (C) Stalks and spores. Wild-type expression patterns of spore specific $(\cot B)$ and stalk specific $(e c m A)$ markers are included for comparison. Arrows depict aberrant $\cot B$ expression within the stalk tube of car4 ${ }^{-}$ fruiting bodies (Only the undifferentiated prespore cells express $\beta$-galactosidase; the terminally differentiated stalk cells do not stain.)

zone yields an apparently enlarged prespore region but, more importantly, prespore cells appear to encroach into the anterior prestalk zone. Anterior cells of $\mathrm{car}^{-}$slugs that express the prespore $\cot B$ marker do not differentiate into stalk cells. Rather, the localized expression of $\cot B$ within the stalk tube of mature fruiting bodies reflects the mispositioning of prespore cells in prestalk regions. We also do not observe mature spore cells in the stalk tube of $\mathrm{car}^{-}$fruiting bodies suggesting that the mislocalized prespore cells in the anterior do not sporulate. This is consistent with our observations that appropriate positioning of prespore cells is required for their terminal differentiation (Richardson et al. 1994). We suggest that CAR4 is required to establish the prestalk/prespore boundary. In addition, because CAR4 expression is enriched in prestalk cells, its absence may cause some cells to divert from a prestalk differentiation pathway into a prespore course. In many car $4^{-}$fruiting bodies the stalk/spore ratio may be reduced when compared with parental controls. However, because $\sim 80 \%$ of wild-type slug cells are prespore, the enhanced expression of prespore markers in car $4^{-}$slugs cannot be explained simply by an alteration in cell type proportions.

The cAMP receptors couple with a variety of intracellular effector systems during early development, and many, if not all, of these are operable during late stages of development (Kimmel and Firtel 1991; Johnson et al. 1992a). One of these downstream effectors is adenylyl cyclase (Konijn et al. 1967; Dinauer et al. 1980; Kimmel and Firtel 1991; Johnson et al. 1992a); thus, the CAR family is atypical as it can directly regulate the production of its own ligand. During early development, gradients of extracellular cAMP mobilize cells for aggregation. Recent data indicate that cells within the slug are likewise chemotactic to extracellular cAMP and suggest that anterior (prestalk) cells exhibit different patterns of motility than do posterior (prespore) cells (Siegert and Weijer 1991, 1992). All four cAMP receptors are present 
during late developmental stages, but there are more cAMP receptors on prestalk than on prespore cells (Schaap and Spek 1984). In addition, CAR1 and CAR3 have significantly greater affinity for cAMP than do CAR2 and CAR4, which show enriched expression in prestalk cells (Johnson et al. 1992b; Saxe et al. 1993; J.M. Louis and A.R. Kimmel, unpubl.). Currently it is not clear whether all of the CAR proteins are linked to identical intracellular signaling pathways, but their differences in affinity for CAMP, in spaciotemporal expression, and (potentially) in downstream signaling cascades must dictate the specificity of the developmental defects of car4 null cells that still express CAR1, CAR2, and/or CAR3 (Saxe et al. 1991b; Johnson et al. 1992b, 1993; Saxe et al. 1993; B. Lee, J.M. Louis, and A.R. Kimmel, in prep.; J.M. Louis and A.R. Kimmel; R. Gollop, and A.R. Kimmel, both unpubl.).

The tips of the aggregation mound and anterior zone of the slug that express CAR4 (and CAR2) maximally are primary sites of cAMP signaling during development (Bonner 1949; MacWilliams 1984; Saxe et al. 1993). These regions behave as classical embryonic organizers, inducing secondary developmental axes in recipient slugs onto which they have been transplanted (Raper 1940; MacWilliams 1984). It is suggested that cAMP gradients emanating from the transplanted exogenous tips are required for these inductive events. Potentially, the absence of CAR4 in the anterior cells of car4- slugs may alter cAMP signaling throughout the slug and the consequent defects in the axial patterning could reflect both autonomous (intracellular) and nonautonomous (extracellular) cellular events. Autonomous cellular responses may result from the intrinsic signal transduction properties of CAR4 itself; nonautonomous functions may require transmembrane signaling that is mediated by CAR1, CAR2 and/or CAR3, but not by CAR4, but is disrupted when extracellular cAMP accumulation is defective in car4 $4^{-}$slugs.

The overexpression of $\cot B$ in $\operatorname{car} 4$ mutants cultured with saturating concentrations of exogenous cAMP indicates that a defect in signal perception, in contrast to an alteration of cAMP production, is at least partially involved in the mutated phenotype. However, it is not clear whether this defect is inherent to the sensory transduction machinery of the $\mathrm{car}^{-}$prespore cells. Prespore gene expression may be modulated through interaction with or signaling by prestalk cells, and resulting defects in the latter population may secondarily alter regulation of the former. This is pivotal as prestalk cells express CAR4 at significantly greater levels than do prespore cells. In addition, preliminary analyses of chimeric wildtype $/ \mathrm{car}^{-}$slugs suggest that $\mathrm{car} 4^{-}$prespore and $\mathrm{car} 4^{-}$ prestalk cells are differentially responsive to the environment provided by wild-type cells (G.T. Ginsburg and A.R. Kimmel, unpubl.).

Although cAMP is a primary signaling molecule only in the Dictyostelids, its cell-surface receptors, the CAR proteins, are members of the superfamily of $G$ proteinlinked receptors (Dohlman et al. 1991). Receptors from various organisms respond to diverse stimuli, including odorants, hormones, neurotransmitters, pheromones, and light but activate common downstream effectors (e.g., adenylyl cyclase, phospholipase $\mathrm{C}$, and ion channels). In Dictyostelium, CAR activation increases the intracellular concentrations of cGMP, DAG, $\mathrm{IP}_{3}, \mathrm{Ca}^{++}$, and $\mathrm{H}^{+}$, in addition to cAMP (Konijn et al. 1967; Dinauer et al. 1980; Europe-Finner and Newell 1987; van Haastert et al. 1989; Milne and Coukell 1991; Kuwayama et al. 1993). CAR-mediated signaling shares homologous regulatory cascades with many G proteinlinked systems, and receptors related to the CAR proteins are required for specific cellular differentiation or proliferation (Dohlman et al. 1991).

The regulation of cell fate by inductive interactions through signaling gradients is recurrent throughout evolution. What has remained elusive are the mechanisms by which short-range or diffusible inductive molecules specify different positional information in an apparent concentration-dependent manner. We suggest that in Dictyostelium regional differences in cAMP concentration gradients, in combination with cell-specific expression of receptors with different affinities for cAMP, direct pattern formation during development. The inability of $c a r 4^{-}$slugs both to produce and to perceive these regionally specific gradients of cAMP may cause defects in patterning and cellular differentiation. It is now essential to analyze the downstream cascades associated with CAR4 signaling to determine how they are ultimately integrated with nuclear events.

\section{Materials and methods}

Growth, development, and DNA-mediated transformation of Dictyostelium

Dictyostelium discoideum wild-type strain NC-4 was grown on bacteria and developed on filters as described previously (Ginsburg and Kimmel 1989). Ax-3 cells, G418-resistant cells and thy $1^{-}$strains were grown in axenic liquid media with or without $20 \mu \mathrm{g} / \mathrm{ml}$ of $\mathrm{G} 418$ or $100 \mu \mathrm{g} / \mathrm{ml}$ of thymidine as required (Dynes and Firtel 1989; Williams et al. 1989; Hadwiger and Firtel 1992; Louis et al. 1993).

Cells were developed on agar plates or nitrocellulose filters at a surface density of $10^{7}$ cells $/ \mathrm{cm}^{2}$ or differentiated in shaking culture at a density of $10^{7}$ cells $/ \mathrm{ml}$ in $10 \mathrm{~mm}$ sodium phosphate (pH 6.4), $2 \mathrm{mM} \mathrm{MgCl}_{2}$, and $0.2 \mathrm{mM} \mathrm{CaCl}$. For cAMP treatment, shaking cultures received $20 \mathrm{nM}$ pulses of cAMP at $6 \mathrm{~min}$ intervals for $5 \mathrm{hr}$ and were subsequently adjusted to and maintained at $300 \mu \mathrm{M}$ cAMP for the remainder of differentiation (Kimmel 1987).

The thy $1^{-}$cell line JH10 was transformed to THY1 as described (Hadwiger and Firtel 1992; Saxe et al. 1993). Greater than $50 \%$ of the transformants had CAR4 disruptions. Cell lines were transformed and selected for G418 resistance as described (Williams et al. 1989; Louis et al. 1993).

\section{Vector constructs}

For expression studies, a full-length CAR4 cDNA was fused in the sense orientation behind the actin 15 promoter in pBS18 (Kumagai et al. 1991).

For homologous recombination, the 3.2-kb BamHI-BamHI fragment containing the entire THY1 gene of Dictyostelium 
was inserted at the end of transmembrane domain seven of CAR4, adjacent to the BstXI site. This site is positioned identically among all of the CAR genes (Saxe et al. 1993).

\section{$\beta$-Galactosidase staining}

Nitrocellulose filters were fixed for $10 \mathrm{~min}$ with a mist of $1 \%$ glutaraldehyde in Z buffer (Richardson et al. 1994). Filters were then treated for an additional 10 min with $0.1 \% \mathrm{NP}-40$ in $\mathrm{Z}$ buffer to permeabilize cells and then rinsed twice in $Z$ buffer before incubation with X-gal staining solution (Dingermann et al. 1989).

\section{Nucleic acid analyses}

Total RNA, poly $(A)^{+}$RNA, or genomic DNA was isolated, size separated by gel electrophoresis, and transferred to nitrocellulose as described (Kimmel and Firtel 1985; Kimmel 1987). Probes were radiolabeled by the random primer method using $\left[\alpha^{-32} \mathrm{P}\right] \mathrm{dCTP}$ and hybridized to blots at $37^{\circ} \mathrm{C}$ in $0.8 \mathrm{M} \mathrm{Na}^{+}, 50 \%$ formamide (Kimmel 1987). Genomic and cDNA libraries were screened at $30^{\circ} \mathrm{C}$ (Saxe et al. 1993). Sequence manipulation and homology assignments (PC-Gene 6.5; Intelligenetics, CA) have been described (Saxe et al. 1993).

\section{cAMP-binding assays}

The binding of $1 \mu \mathrm{M}$ radiolabeled cAMP to the surface of cells was preformed in the presence of saturated ammonium sulfate buffer (Kimmel 1987). Competition with $1 \mathrm{~mm}$ unlabeled cAMP was performed in parallel. cAMP binding sites were expressed on a per cell basis.

\section{Acknowledgments}

We are very grateful to R.A. Firtel, W. Loomis, D. Ratner and J.G. Williams for their generous supply of reagents. We also appreciate the extremely helpful discussions and comments of P. Balint-Kurti, P.N. Devreotes, J. Kennison, D. Richardson, K. Rogers, P.L. Schwartzberg, and R.T Simpson.

The publication costs of this article were defrayed in part by payment of page charges. This article must therefore be hereby marked "advertisement" in accordance with 18 USC section 1734 solely to indicate this fact.

\section{References}

Bonner, J.T. 1949. The demonstration of acrasin in the later stages of development of the slime mold Dictyostelium discoideum. J. Exp. Zool. 110: 259-271.

Dinauer, M.C., T.L. Steck, and P.N. Devreotes. 1980. Cyclic $3^{\prime}, 5^{\prime}$-AMP relay in Dictyostelium discoideum. Adaptation of the cAMP signaling response during cAMP stimulation. $J$. Biol. Chem. 86: 554-561.

Dingermann, T., N. Reindl, H. Werner, M. Hildebrandt, W. Nellen, A. Harwood, J. Williams, and K. Nerke. 1989. Optimization and in situ detection of Escherichia coli beta-galactosidase gene expression in Dictyostelium discoideum. Gene 85: 353-362.

Dohlman, H.G., J. Thorner, M.G. Caron, and R.J. Lefkowitz. 1991. Model systems for the study of seven-transmembranesegment receptors. Annu. Rev. Biochem. 60: 653-688.

Dynes, J.L. and R.A. Firtel. 1989. Molecular complementation of a genetic marker in Dictyostelium using a genomic DNA library. Proc. Nat1. Acad. Sci. 86: 7966-7970.
Early, A.E., M.J. Gaskell, D. Traynor, and J.G. Williams. 1993. Two distinct populations of prestalk cells within the tip of the migratory Dictyostelium slug with differing fates at culmination. Development 1218: 353-362.

Esch, R.K. and R.A. Firtel. 1991. cAMP and cell sorting control the spatial expression of a developmentally essential celltype-specific ras gene in Dictyostelium. Genes \& Dev. 5: 921.

Europe-Finner, G. and P. Newell. 1987. Cyclic AMP stimulates accumulation of inositol trisphosphate in Dictyostelium. I. Cell Sci. 87: 221-229.

Fosnaugh, K.L. and W.F. Loomis. 1993. Enhancer regions responsible for temporal and cell-type-specific expression of a spore coat gene in Dictyostelium. Dev. Biol. 157: 38-48.

Gaskell, M.J., K.A. Jermyn, D.J. Watts, T. Treffry, and J.G. Williams. 1992. Immuno-localization and separation of multiple prestalk cell types in Dictyostelium. Differentiation 51: 171-176.

Ginsburg, G. and A.R. Kimmel. 1989. Inositol trisphosphate and diacylglycerol can differentially modulate gene expression in Dictyostelium. Proc. Nat1. Acad. Sci. 86: 9332-9336.

Gomer, R., D. Armstrong, B. Leichtling, and R. Firtel. 1986. cAMP induction of prespore and prestalk gene expression in Dictyostelium is mediated by the cell surface cAMP receptor. Proc. Natl. Acad. Sci. 83: 8624-8628.

Hadwiger, J.A. and R.A. Firtel. 1992. Analysis of $\mathrm{G} \alpha_{4}$, a G-protein subunit required for multicellular development in Dictyostelium. Genes \& Dev. 6: 38-49.

Haribabu, B. and R. Dottin. 1986. Pharmacological characterization of cyclic AMP receptors mediating gene-regulation in Dictyostelium discoideum. Mol. Cell. Biol. 6: 2402-2408.

Hereld, D., R. Vaughan, J.Y. Kim, J. Borleis, and P. Devreotes. 1994. Localization of ligand-induced phosphorylation sites to serine clusters in the C-terminal domain of the Dictyostelium cAMP receptor, cAR1. J. Biol. Chem. 269: 70367044.

Jermyn, K., M. Berks, R. Kay, and J. Williams. 1987. Two distinct classes of prestalk-enriched messenger RNA sequences in Dictyostelium discoideum. Development 100: 745-755.

Johnson, R.L., R. Gunderson, D. Herald, G.S. Pitt, S. Tugendreich, C.L. Saxe, A.R. Kimmel, and P.N. Devreotes. 1992a. G-protein signaling pathways mediate development in Dictyostelium. Cold Spring Harbor Symp. Quant. Biol. 57: 169-176.

Johnson, R.L., P.J. van Haastert, A.R. Kimmel, C.L. Saxe, B. Jastorff, and P.N. Devreotes. 1992b. The cyclic nucleotide specificity of three cAMP receptors in Dictyostelium. I. Biol. Chem. 267: 4600-4607.

Johnson, R.L., C.L. Saxe, R. Gollop, A.R. Kimmel, and P.N. Devreotes. 1993. Identification and targeted gene disruption of cAR3, a cAMP receptor subtype expressed during multicellular stages of Dictyostelium development. Genes \& Dev. 7: 273-282.

Kimmel, A.R. 1987. Different molecular mechanisms for cAMP regulation of gene expression during Dictyostelium development. Dev. Biol. 122: 163-171.

Kimmel, A.R. and R.A. Firtel. 1985. Sequence organization and developmental expression of an interspersed, repetitive element and associated single-copy DNA sequences in Dictyostelium discoideum. Mol. Cell. Biol. 5: 2123-2130.

- 1991. cAMP signal transduction pathways regulating development of Dictyostelium discoideum. Curr. Opin. Genet. Dev. 1: 383-390.

Klein, P., A. Theibert, D. Fontana, and P.N. Devreotes. 1985. Identification and cyclic AMP induced modification of the cyclic AMP receptor in Dictyostelium discoideum. I. Biol. 
Chem. 260: 1757-1764.

Klein, P., T. Sun, C.L. Saxe, A.R. Kimmel, R. Johnson, and P.N. Devreotes. 1988. A chemoattractant receptor controls development in Dictyostelium discoideum. Science 241: 14671472.

Konijn, T.M., J.G.C. Van de Meene, J.T. Bonner, and D.S. Barkley. 1967. The acrasin activity of adenosine-3',5'-cyclic phosphate. Proc. Nat1. Acad. Sci. 58: 1152-1154.

Kumagai, A.M., J.A. Hadwiger, M. Pupillo, and R.A. Firtel. 1991. Molecular analysis of two $\mathrm{G} \alpha$ protein subunits of Dictyostelium. J. Biol. Chem. 266: 1220-1228.

Kuwayama, H., S. Ishida, and P.J.M. van Haastert. 1993. Nonchemotactic Dictyostelium discoideum mutants with altered cGMP signal transduction. I. Cell Biol. 123: 14531462.

Louis, J.M., C.L. Saxe, and A.R. Kimmel. 1993. Two transmembrane signaling mechanisms control expression of the cAMP receptor gene CARl during Dictyostelium development. Proc. Natl. Acad. Sci. 90: 5969-5973.

MacWilliams, H.K. 1984. Cell-type ratio and shape in slugs of the cellular slime molds. In Pattern formation: A primer in developmental biology (ed. M. Maclacinski and S.V. Bryant), pp. 127-162. MacMillan Pess, New York.

Mann, S.K. and R.A. Firtel. 1991. A developmentally regulated, putative serine/threonine protein kinase is essential for development in Dictyostelium. Mech. Dev. 35: 89-101.

Milne, J.L. and M.B. Coukell. 1991. A Ca ${ }^{++}$transport system associated with the plasma membrane of Dictyostelium discoideum is activated by different chemoattractant receptors. J. Cell Biol. 112: 103-10.

Oyama, M. and D. Blumberg. 1986. Interaction of cAMP with the cell surface receptor induces cell type specific messenger RNA accumulation in Dictyostelium discoideum. Proc. Natl. Acad. Sci. 83: 4819-4823.

Raper, K. 1940. Pseudoplasmodium formation and organisation in Dictyostelium discoideum. I. Elisha Mitchell Sci. Soc. 59: 241-282.

Ratner, D. and W. Borth. 1983. Comparison of differentiating Dictyostelium discoideum cell-types separated by an improved method of density gradient centrifugation. Exp. Cell Res. 143: $1-13$.

Richardson. D., W.F. Loomis, and A.R. Kimmel. 1994. Progression of an inductive signal activates sporulation in Dictyostelium discoideum. Development (in press).

Saxe, C.L., R.L. Johnson, P.N. Devreotes, and A.R. Kimmel. 1991a. Expression of a cAMP receptor gene of Dictyostelium and evidence for a multigene family. Genes \& Dev. 5: 1-8. 1991b. Multiple genes for cell surface cAMP receptors in Dictyostelium discoideum. Dev. Genet. 12: 6-13.

Saxe, C.L., G.T. Ginsburg, J.M. Louis, R. Johnson, P.N. Devreotes, and A.R. Kimmel. 1993. CAR2, a prestalk cAMP receptor required for normal tip formation and late development of Dictyostelium discoideum. Genes \& Dev. 7: 262272.

Schaap, P. and W. Spek. 1984. Cyclic AMP binding to the cell surface during development of Dictyostelium discoideum. Differentiation 27: 83-87.

Schaap, P. and R. Van Driel. 1985. Induction of post-aggregative differentiation in Dictyostelium discoideum by cAMP. Exp. Cell Res. 159: 388-398.

Shaw, D.R., H. Richter, R. Giorda, T. Ohmachi, and H.L. Ennis. 1989. Nucleotide sequences of Dictyostelium discoideum developmentally regulated cDNAs rich in (AAC) imply proteins that contain clusters of asparagine, glutamine, or threonine. Mol. Gen. Genet. 218: 453-459.

Siegert, F. and C.J. Weijer. 1991. Analysis of optical density wave propagation and cell movement in the cellular slime mould Dictyostelium discoideum. Physica D. 49: 224-232. . 1992. Three-dimensional scroll waves organize Dictyostelium slugs. Proc. Natl. Acad. Sci. 89: 6433-6437.

Sternfeld, J. and C. David. 1982. Fate and regulation of anteriorlike cells in Dictyostelium slugs. Dev. Biol. 93: 111-118.

Sun, T.J. and P.N. Devreotes. 1991. Gene targeting of the aggregation stage cAMP receptor cARl in Dictyostelium. Genes \& Dev. 5: 572-582.

van Haastert, P., M. de Vries, L. Penning, E. Roovers, J. van der kaay, C. Erneux, and M.M. van Lookern Campagne. 1989. Chemoattractant and guanosine $5^{\prime}$-[-thio|triphosphate induce the accumulation of inositol 1,4,5-trisphosphate in Dictyostelium cells that are labeled with $\left[{ }^{3} \mathrm{H}\right]$ inositol by electroporation. Biochem. J. 258: 577-586.

Williams, J.G. 1991. Spatial regulation during Dictyostelium development. Curr. Opin. Genet. Dev. 1: 358-362.

Williams, J.G., K.T. Duffy, D.P. Lane, S.J. McRobbie, A.J. Harwood, D. Traynor, R.R. Kay, and K.A. Jermyn. 1989. Origins of the prestalk-prespore pattern in Dictyostelium development. Cell 59: 1157-1163. 


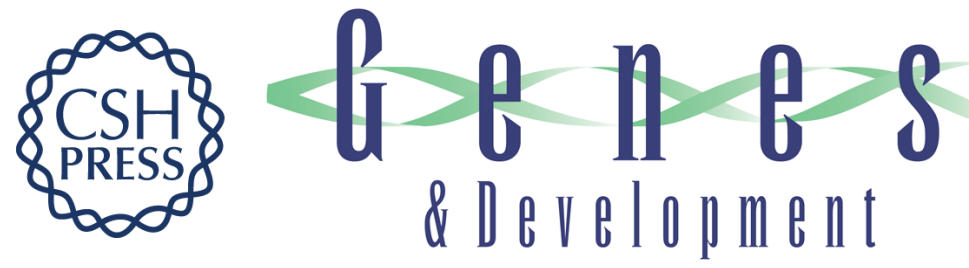

\section{The cAMP receptor CAR4 regulates axial patterning and cellular differentiation during late development of Dictyostelium.}

J M Louis, G T Ginsburg and A R Kimmel

Genes Dev. 1994, 8:

Access the most recent version at doi:10.1101/gad.8.17.2086

References This article cites 46 articles, 23 of which can be accessed free at:

http://genesdev.cshlp.org/content/8/17/2086.full.html\#ref-list-1

License

Email Alerting

Service

Receive free email alerts when new articles cite this article - sign up in the box at the top right corner of the article or click here.

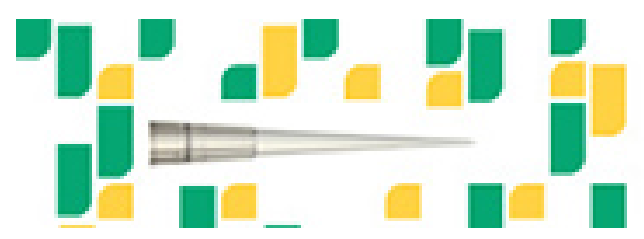

Focused on your science. 